\title{
The analysis of the long-term outcomes in elderly women treated for locally advanced cervical cancer
}

\author{
Krzysztof Koper, MD, PhD $12^{*}$, Konrad Dziobek, MD, PhD ${ }^{3.4^{*}}$, Prof. Roman Makarewicz, MD, PhD 5.6, \\ Joanna Terlikiewicz, MD, PhD5 , Prof. Magdalena Dutsch-Wicherek, MD, PhD' \\ 'Department of Oncology, Radiotherapy and Oncological Gynecology, Nicolaus Copernicus University in Torun, Ludwik Rydygier Collegium \\ Medicum in Bydgoszcz, ${ }^{2}$ Department of Chemotherapy, Franciszek Lukaszczyk Oncology Centre, Bydgoszcz, ${ }^{3}$ Department of Oncological \\ Gynecology. Franciszek Lukaszczyk Oncology Centre, Bydgoszcz, ${ }^{4}$ Oncological Gynecology Department, The Maria Sklodowska-Curie \\ Memorial Cancer Centre and Institute of Oncology, Krakow Branch, Krakow, ${ }^{5}$ Department of Brachytherapy, Franciszek Lukaszczyk Oncology \\ Centre, Bydgoszcz, ${ }^{6}$ Department of Oncology and Brachytherapy, Nicolaus Copernicus University in Torun, Ludwik Rydygier Collegium \\ Medicum in Bydgoszcz, Poland \\ *The first two authors contributed equally in this work.
}

\begin{abstract}
Purpose: Locally advanced cervical cancer (LACC) should be treated with a combination of external irradiation and brachytherapy with concurrent chemotherapy. However, as cervical carcinoma cells can disperse by way of the lymphatic system to either pelvic or para-aortic nodes, planning the extent of radiation requires precise information about the spread of the disease to the lymph nodes, especially to the para-aortic area.

Material and methods: All of the 75 women included in our study underwent chemoradiotherapy, which started with brachytherapy. Out of them, 54 have undergone radical hysterectomy with lymphadenectomy followed by chemoradiation. We have retrospectively analyzed the 5-year overall survival (OS) rates relative to the lymph node involvement, the type of lymphadenectomy performed (pelvic, para-aortic, or both), the size of the tumor $(>$ or $<4 \mathrm{~cm}$ ), the histological type, grading, and the age of patients.

Results: We observed significant differences in the OS rates relative to the age of the patients with LACC. We noted significant differences in the OS rates related to para-aortic lymphadenectomy and presence of lymph node metastases.

Conclusions: Para-aortic lymphadenectomy seems to have a positive influence on long-term outcomes in the LACC patients, and elderly patients may benefit more from applied therapy.

Key words: brachytherapy, chemoradiotherapy, locally advanced cervical cancer, lymphadenectomy.

\section{Purpose}

In some countries such as Poland, a national screening program for cervical cancer has not yet been instituted. Most patients are therefore still diagnosed with locally advanced stages of cervical cancer (LACC), and sometimes even with more widespread forms of the disease. Consequently, a proper treatment options for patients with LACC constitute a serious clinical problem.

Historically, the migration of people from Europe to America at the end of $19^{\text {th }}$ century was linked with an increased incidence of cervical cancer, and this is easily understood when the risk factors for this disease are considered. At that time, cervical cancer therapy was developed. Nowadays, the problem of people migrating to Europe has create the debate about proper treatment for LACC even more critical.
Locally advanced cervical cancer, including stage IB2 on the FIGO (the International Federation of Gynecology and Obstetrics) classification, should be treated with a combination of external irradiation and brachytherapy with concurrent chemotherapy. This strategy is considered the standard treatment for bulky cervical cancer in both Europe and North America. This approach is routinely used in Poland. However, as cervical carcinoma cells can disperse by way of the lymphatic system to either the pelvic or para-aortic nodes, planning the extent of radiation requires precise information about the spread of the disease to the lymph nodes, especially to the para-aortic area. Since the poor prognosis is associated with para-aortic involvement in cervical cancer [1,2], it would seem that a treatment strategy consisting of para-aortic lymphadenectomy followed by chemoradiation with an extended field of radiotherapy is linked with the best
Address for correspondence: Krzysztof Koper, MD, PhD, Department of Oncology, Radiotherapy and Oncological Gynecology, Nicolaus Copernicus University in Torun, Ludwik Rydygier Collegium Medicum in Bydgoszcz, 2 Romanowskiej Str., 85-796 Poland, phone: +48 5237433 44,

๑ e-mail: krzyskoper@gmail.com
Received: 28.09.2018

Accepted: 05.10 .2018

Published: 30.10 .2018 
long-term outcomes. The para-aortic lymph node status in pretreatment staging may be conducted using two kind of basic staging strategies. One is the performance of para-aortic lymphadenectomy and histopathological verifications, and the other is the use of imaging such as positron emission tomography (PET), magnetic resonance imaging (MRI), and computed tomography (CT). Generally, surgery facilitates the pretreatment staging. Due to high number (about $20-50 \%$ ) of false negative rates generated, the ubiquitous use of CT and NMR remains unsatisfactory in detecting nodal involvement [3], attracting more attention on PET or surgical staging. Chantalat et al. have pointed out that para-aortic lymph node involvement is more often associated with pelvic node involvement, as approximately $70 \%$ of all patients with positive para-aortic lymph nodes also have pelvic lymph node metastases. They compared the long-term survival rates of the patients treated with chemoradiation followed by surgical staging of the para-aortic area and by staging based on PET imaging. They observed better survival outcomes in the group of patients treated surgically [1,2], though the difference was not statistically significant. In fact, no randomized trial has validated PET scan as a feasible alternative to surgical pretreatment staging for para-aortic lymph nodes in LACC. Surgical staging is highly specific for detecting lymph node metastases [4]. Furthermore, as various studies have demonstrated, surgery may be usefully applied in treating patients with LACC. In a group of cervical cancer patients with FIGO stage IB2 to IIA2, Dereks et al. observed that the 5-year overall survival (OS) rate as $83 \%$, and the disease-free survival (DFS) rate as $82 \%$ when treated with the Wertheim-Okabayashi surgical procedure followed by adjuvant radiotherapy and chemoradiotherapy (50\% of cases) [5]. Radical hysterectomy with pelvic lymph node dissection and primary chemoradiotherapy have proven equally effective in treating bulky early stage cervical cancer (stages IB2 to IIA2); therefore, in most oncology centers, the indications for the Wertheim procedure are limited to IB1 cases. Nevertheless, the use of para-aortic lymph node assessment by imaging or surgical staging is helpful in tailoring ra-

Table 1. Clinical characteristics of the patients included in the study

\begin{tabular}{lcc}
$\begin{array}{l}\text { Clinical } \\
\text { parameters }\end{array}$ & $\begin{array}{c}\text { Age }<62 \text { years } \\
(n=53)\end{array}$ & $\begin{array}{c}\text { Age } \geq 62 \text { years } \\
(n=22)\end{array}$ \\
\hline Grade & 0 & $1(5 \%)$ \\
\hline G1 & $41(77 \%)$ & $17(77 \%)$ \\
\hline G2 & $12(23 \%)$ & $4(18 \%)$ \\
\hline G3 & & \\
\hline IGO & $20(38 \%)$ & $9(41 \%)$ \\
\hline IIA and IIB & $17(32 \%)$ & $8(36 \%)$ \\
\hline IIIA and IIIB & $11(20 \%)$ & $3(14 \%)$ \\
\hline IVA & $5(10 \%)$ & $2(9 \%)$
\end{tabular}

diotherapy. When positive para-aortic nodes are found, the radiation field may be extended from the pelvis to the para-aortic area. For our study, we decided to retrospectively analyze those cases of LACC treated surgically followed by chemoradiation, according to the range (extent) of lymphadenectomy. Since para-aortic node involvement is associated with poor survival outcomes [1] (in $17 \%$ of cases, the para-aortic area is the place of progression or relapse in cervical cancer), the goal of our study was to examine whether the extent of surgery may influence long-term outcomes. We wanted to investigate whether the value of para-aortic lymphadenectomy was a therapeutic value or simply diagnostic.

\section{Material and methods}

\section{Patients}

At the Lukaszczyk Oncological Center in Bydgoszcz, we retrospectively evaluated a series of women who had been treated between 2008 and 2013 for cervical carcinoma for LACC, up to and including stage IB2 on the FIGO classification. All of the 75 the women included in our study, usually had undergone a chemoradiotherapy followed by brachytherapy. Fifty-four of them have had radical hysterectomy with lymphadenectomy followed by chemoradiation. Adjuvant chemoradiotherapy had been used for cases with high - risk prognostic factors, including tumor size $(>4 \mathrm{~cm})$, parametrial invasion, and lymph node metastasis. Radiotherapy was performed using whole pelvis external beam radiation (50.4 Gy in 28 fractions) and chemotherapy consisted of a platinum - based regimen administered in four to six cycles. The database of the Kujawsko-Pomorski regional office of the National Health System of Poland provided the data on overall survival rates. Lukaszczyk Oncological Center is a referring oncology center for the Kujawsko-Pomorski region in Poland, which has 2.3 million habitants. We have retrospectively analyzed the 5-year OS rates regarding to various clinical parameters as follows to lymph node involvement, the kind of lymphadenectomy (pelvic, para-aortic, or both) performed, tumor size ( $>$ or $<4 \mathrm{~cm}$ ), histological type, grading, and age of the patients.

Patients who underwent lymphadenectomies had had systemic lymphadenectomies consisting of pelvic lymph nodes dissection (external iliac nodes, internal iliac nodes, common iliac nodes). However, in beginning of 2010, the para-aortic lymph node resections were routinely applied. The para-aortic lymphadenectomy was performed up to the inferior mesenteric artery. The study was approved by the Bioethics Committee of the Collegium Medicum Nicolaus Copernicus University in Bydgoszcz (Table 1).

\section{Statistical analysis}

Data analysis was performed using STATISTICA version 13.0. Characteristics were compared between groups with the Chi-squared test for qualitative, nominal, and dichotomous variables, and the Mann-Whitney $U$ test for quantitative variables. Survival was evaluated using the Kaplan-Meyer method. Groups were compared 
using log-rank test and a multivariate analysis was conducted using Cox's model. The results were analyzed and the end points such as: death or the last visit in the hospital, which data was available in the medical reports. The differences were considered statistically significant at $p<0.05$. The parameters were also expressed as median and range.

\section{Results}

\section{Lymph node status analysis}

Lymphadenectomy was performed in all the patients operated and included in the study; in 54 patients, both pelvic and para-aortic lymphadenectomies were performed. We removed 1,205 nodes from the pelvic area (range: 1-52), and 232 from the para-aortic area (range: 1-18) (Table 2).

\section{Overall survival and disease-free survival analysis}

We assessed the 5-year OS rate in each patient, taking into account the following clinical features: lymph node involvement, the kind of lymphadenectomy (pelvic, paraaortic, or both) performed, tumor size ( $>$ or $<4 \mathrm{~cm}$ ), histological type, and grading. Furthermore, using multivariate Cox analysis, we have calculated the predictive value of the appearance of these features and their impact on long-term outcomes. We observed significant differences
Table 2. The number of patients with pelvic lymphadenectomy and with both pelvic and paraaortic lymphadenectomies

\begin{tabular}{lc} 
Pelvic lymphadenectomy & $54(100 \%)$ \\
\hline Pelvic NO & $37(69.5 \%)$ \\
\hline Pelvic N1 & $17(30.5 \%)$ \\
\hline Para-aortic lymphadenectomy & $36(65 \%)$ \\
\hline Para-aortic NO & $27(76 \%)$ \\
\hline Para-aortic N1 & $9(24 \%)$
\end{tabular}

in OS rates relative to para-aortic lymphadenectomy and presence of lymph node metastases (Table 3).

We observed significant differences in OS rates relative to the age of the patients in the whole group of patients treated only by chemoradiation and by surgery as well as adjuvant chemoradiation (Figure 1).

\section{Discussion}

The histopathological parameters for typing the tumor in the studies on LACC varied. The published ranges according to tumor grade were as follows: G1 in 4\% [6], $10 \%$ abdominal radical hysterectomy $(\mathrm{ARH})$, and $7 \%$ robotic radical hysterectomy (RRH) [7] of cases; G2 in 70\% [6], 40\% ARH [7], and 42\% RRH [7] of cases; G1-G2 in 66\%

Table 3. Influence of followed clinical parameters: lymph nodes status, histological type, grading, tumor size, and range of lymphadenectomy on overall survival (OS) in multivariate Cox analysis

\begin{tabular}{|c|c|c|c|c|}
\hline Clinical features & No. of patients & Hazard ratio & $95 \% \mathrm{Cl}$ & $p$ \\
\hline \multicolumn{5}{|l|}{ Lymph node status } \\
\hline NO & 32 & Reference & & \\
\hline N1 (both groups) & 21 & 1.16 & $0.50-2.27$ & 0.65 \\
\hline N1 (pelvic) & 17 & 0.96 & $0.47-1.93$ & 0.91 \\
\hline N1 (para-aortic) & 9 & 2.7 & $0.98-7.44$ & 0.05 \\
\hline \multicolumn{5}{|l|}{ Histological type } \\
\hline Squamous & 46 & Reference & & \\
\hline Adenocarcinoma & 8 & 0.96 & $0.29-3.14$ & 0.95 \\
\hline \multicolumn{5}{|l|}{ Grade } \\
\hline $\mathrm{G} 1+\mathrm{G} 2$ & 38 & Reference & & \\
\hline G3 & 16 & 0.97 & $0.46-2.04$ & 0.93 \\
\hline \multicolumn{5}{|l|}{ Tumor size } \\
\hline Less than $4 \mathrm{~cm}$ & 39 & Reference & & \\
\hline Greater than $4 \mathrm{~cm}$ & 15 & 1.06 & $0.37-3.07$ & 0.9 \\
\hline \multicolumn{5}{|l|}{ Site of lymphadenectomy } \\
\hline Only pelvic lymphadenectomy & 18 & Reference & & \\
\hline $\begin{array}{l}\text { Pelvic and para-aortic } \\
\text { lymphadenectomy }\end{array}$ & 36 & 0.52 & $0.28-0.98$ & 0.04 \\
\hline
\end{tabular}




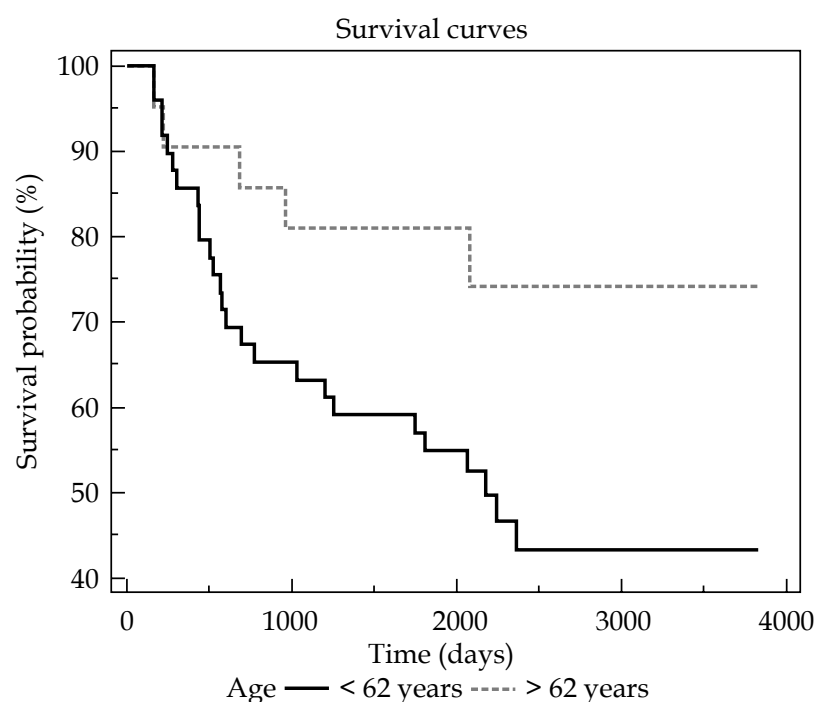

Fig. 1. Kaplan-Meier curve (OS) depending on the age of patients treated with chemoradiotherapy (starting from brachytherapy)

[8] of cases; G3 in 25\% [6], 44\% ARH [7], 42\% RRH [7], and $34 \%$ [8] of cases. In our study, we have found grade G1, G2 (both $76 \%$ ), and G3 in $24 \%$ patients.

Lymphatic space involvement (LSI) was found in $17 \%$ [6], 31\% ARH [7], 27\% RRH [7], and 34\% [8] of cases, whereas in $35 \%$ of patients with LACC, we found lymphatic vascular space invasion (LVSI). Typically, LVSI is linked with a greater number of lymph node metastases and is often presented in multivariate Cox analysis as a negative prognostic factor $[7,8]$. However, Dereks et al. concluded that the presence of either LVSI or lymph node metastases was not associated with a poor prognosis [5] In our study, LVSI invasion did not influence OS.

MacDonald et al. presented analysis of 4,559 radical hysterectomy and lymphadenectomy patients. In this group of patients, $18.6 \%$ had lymph node metastases. The incidence of lymph node metastases increased in relation to the FIGO stage. For example, the para-aortic area was involved in $0.7 \%$ of cases in stage I, but in $5 \%$ of cases in stage II [9]. In LACC patients, the incidence of lymph node metastases is significant, and pretreatment staging requires precise information about the involvement of the node area by neoplasm in order to plan the appropriate adjuvant therapy (extent of radiotherapy). In LACC patients, the incidence of para-aortic lymph node metastases ranges from $5 \%$ to $33 \%[10,11]$. In their study, which included patients with LACC in FIGO stage IB2 to IIIB, Del Pino et al. found lymph node metastases in $21.2 \%$ of cases. In IB2 (only 12 patients), the authors did not observe para-aortic metastases and in 33\% of cases, the pelvic nodes were positive. More advanced FIGO stage disease was related to an increased number of positive para-aortic as well as pelvic nodes. Retrospectively, in patients with stage IIB, $17 \%$ had positive pelvic nodes and $25.5 \%$ positive para-aortic nodes. Furthermore, in patients with stage IIIB, 33\% had positive nodes in the pelvic region and $43.5 \%$ in the para-aortic region [12]. Zand et al. prospectively examined patients with LACC [13];
$22 \%$ of them had para-aortic metastases. In other studies, para-aortic lymph node metastases were observed in $11 \%$ [4], 14\% [14], 15\% [15], and 15.8\% [16] of LACC patients. Leblanc et al. examined the group of 196 patients derived from two different oncology centers, on whom para-aortic lymphadenectomy had been performed and suggested that in cervical cancer patients, the procedure should be restricted to the lymph nodes located below the IMA (interior mesenteric artery). Only in one case, the lymph node metastases were located in the supra mesenteric area [15]. By contrast, Gil-Moreno et al. have revealed that in $30 \%$ of cases, infrarenal positive nodes may be found even among negative inferior mesenteric lymph nodes [17]. The presented results of the lymph node metastasis range revealed that these problems need further investigation, and that adjuvant therapy of LACC patients requires a proper assessment of the para-aortic area, since systemic complete lymphadenectomy in cervical cancer patients enables planning adjuvant therapy. In women with cervical cancer in stages IB and II, the removal of bulky positive nodes during the surgery has a curative potential as well. Morice et al. revealed that the 3-year OS rate in N1 patients was $55 \%$, which was significantly lower than in N0 patients (94\%). When the node metastases were located in the pelvis, the 3-year OS rate was 59\% in unilateral metastases and $47 \%$ in bilateral metastases. However, para-aortic lymph node involvement was associated with a diminished 3-year OS rate in the range of $32 \%$. A prognostic factor in multivariate analysis that influenced OS rate was age (less than 30 years, with tumor size greater than $4 \mathrm{~cm}$, and positive lymph nodes) [18]. Dereks et al. demonstrated that tumor diameters and histology were independent predictors of survival in stage IB2 of cervical cancer [5]. In your study, only para-aortic lymphadenectomy and presence of lymph node metastases influenced the multivariate analysis of OS. Furthermore, they indicated that the number of positive lymph nodes was linked with a worse prognosis [9]. Overall survival significantly decreased the percentage of the number of nodes involved and was the worst when there were more than 10 positive lymph nodes. In most cases, node-positive disease was also associated with a tumor larger than $4 \mathrm{~cm}$. The 5-year OS rate was 39\% for patients with positive para-aortic lymph nodes and was significantly better when only the pelvic nodes were involved (OS rate in this group ranges to $67 \%$ ). Using multivariate analysis, MacDonald et al. have demonstrated that squamous cell histology is linked with better long-term survival than adenocarcinoma histology. While analyzing the common influence for OS histology and lymph node involvement, the authors pointed out converse observations. When lymph nodes are involved, squamous cell histology has a better overall survival rate than adenocarcinoma histology. However, when no lymph node disease is observed, adenocarcinoma histology is linked with a better long-term OS rate [9]. We have not, however, observed the differences in OS rates in relation to histology.

In another study, Cosin et al. presented their 10-year observation on the OS rates of 266 patients with cervi- 
cal carcinoma who underwent extraperitoneal pelvic and para-aortic lymphadenectomy prior to radiotherapy. The authors pointed out that the resection of bulky nodes increased long-term survival [19]. Moreover, Goff et al. observed the 5-year OS rate in patients without lymph node metastases to be $74 \%$ [14]. However, when positive nodes were found, the OS rate was $58 \%$ for those with microscopic nodal involvement and 39\% for those with macroscopic involvement. Macroscopic involvement was closely related to worse long-term outcomes. Le Blanc et al. presented results showing that the 5-year OS rate for women with metastases in the para-aortic area was $52 \%$. The number of positive nodes was a significant prognostic variable, and the overall 5 -year survival rate was $58.3 \%$ $[15,20]$. Furthermore, the authors observed that complete surgical removal of positive lymph nodes correlated with a survival advantage. In our own study using the multivariate Cox analysis, we observed the link between para-aortic lymph node removal and better 5 -year OS rates.

In contrast to the observations presented above, del Pino et al. have suggested that the removal of nodes has no therapeutic benefit [12]. In the group of patients with para-aortic positive nodes, complete removal of nodes was not associated with a higher mortality risk than para-aortic node sampling alone, although the univariate Cox logistic model indicates that lymph node metastases in both the pelvic and para-aortic areas are associated with an increased risk of death. The authors concluded that evaluation of lymph node status in the para-aortic area may provide valuable information about mortality risk in LACC patients. While patients with positive nodes, regardless of their location (pelvic or para-aortic), have worse long-term survival rates than patients without metastases (N0), del Pino et al. have observed no difference in OS and DFS rates between the patients with metastases in the pelvic as opposed to the para-aortic nodes. Overall, the presence of lymph node metastases is a relevant prognostic factor in patient with LACC [12].

The therapeutic value of complete lymphadenectomy, as presented above, remains controversial. Some authors recommend irradiation when suspicious bulky nodes are found; others recommend extensive node resection, e.g., laterally extended endopelvic resection (LEER), because radiotherapy alone is not reliable for the sterilization of bulky positive nodes. Maurice $e t$ al. have pointed out that removing positive lymph nodes from the para-aortic area does not increase the preoperative morbidity in comparison to treatment consisting of radiotherapy and surgery without para-aortic node removal [18]. Similarly, Gaff et al. have demonstrated a survival advantage for women who undergo surgical staging [14].

In most cases in the presented studies, the lymphadenectomies were performed as an extraperitoneal laparoscopy, but this procedure still involves a certain degree of morbidity. While the range of complication is smaller than for open techniques, para-aortic lymphadenectomies may be linked with the following severe complications: deep vein thrombosis, bowel obstruction, hematoma, and retroperitoneal abscess. These surgical procedures may also evoke distant morbidity, such as urinary inconti- nences, ureter stenosis, lymphedemas, leg lymphedemas, and vesicovaginal fistula. The authors observed complications in $7.5 \%$ of cases. However, none of them delayed curative treatment $[15,20]$. Lymphocele is not a severe complication of lymphadenectomy and should only be treated if symptomatic. Asymptomatic lymphocele appears in about $5-6 \%$ of lymphadenectomies performed for gynecological malignancy and warrants only observation [21]. Surgical staging in patients with LACC neither influences nor delays adjuvant therapy. The choice of surgery to treat LACC is linked with complications related to radical hysterectomy, not just to lymphadenectomy. Radical hysterectomy-related complications differ depending on the surgical technique involved, such as ARH or RRH, and include: post-operative transfusion ARH 10\%, RRH 3\% [7], infections ARH 4\%, RRH 2\% [7], ileus ARH 1\%, RRH 0\% [7], ureteral fistula ARH 2.5\%, RRH 1\% [7], lymphocyst ARH 10.5\%, RRH 3.5\% [7], vaginal dehiscence ARH 0.7\%, and RRH 3.9\% [7].

Additionally, due to the morbidity associated with the surgical procedure and the frequent incidence of vascular anomalies in the para-aortic area requiring an extensive experience of the gynecologist, some authors have proposed that para-aortic lymph node resection in cervical cancer should be limited to those patients with positive pelvic nodes. However, no increased morbidity related to the surgical procedure was generally observed [6]. Overall, the level of peri-operative morbidity is acceptable, as confirmed in a randomized trial [6]. Furthermore, this would seem to indicate that para-aortic lymphadenectomy can be applied as a routine procedure performed in all cervical cancer cases by all practicing gynecological oncologists.

The range of para-aortic lymphadenectomies is still a matter of debate among gynecological oncologists. The pretreatment staging requires the dissection of para-aortic lymph nodes, but different authors propose various ranges for para-aortic lymphadenectomies. Staging is essential to plan the extent of the radiotherapy. Most surgeons established the necessity of removing the lymph nodes from the infra mesenteric area, but more extensive lymphadenectomies up to the ovarian vessel or left renal vein were also proposed. Outlander in meta-analysis revealed para-aortic metastases in $1 \%$ of cases and only $0.25 \%$ located above the IMA. Para-aortic lymph node metastases are rare in the superior mesenteric area, in the absence of positive nodes below the IMA. Applying this therapeutic strategy, in our study, we routinely performed the para-aortic lymphadenectomy up to the IMA. The different authors presented different necessary ranges for para-aortic and pelvic lymphadenectomies. The median numbers of harvested pelvic nodes were 19 [6], 29 [18], and 20 [9]; the median numbers of harvested para-aortic nodes were 17 [6], 22 [15,20], and 28.5 [16]. In our group of patents, the median numbers of harvested pelvic nodes were 23 and para-aortic was 13 .

The sensitivity of CT and MRI in diagnosing lymph node metastases is in the range of $60 \%$. Lymphadenectomy appears to be an appropriate staging procedure with prognostic value. However, when more advanced imag- 
ing techniques are used, only the MRI results of pretreatment staging are superior. Lee et al. compared two therapeutic strategies applied in patients with cervical cancer in FIGO stage IB. The first was radical surgery as the primary procedure (followed by chemoradiation); the second was chemoradiation alone with the therapeutic schema based on MRI. This was followed later by an analysis of risk factors, and then the choice between primary surgery or chemoradiation (radiotherapy combined with chemotherapy RCTX, extended to encompass the para-aortic region). This strategy of risk factor analysis was more cost-effective than either primary surgery or primary chemoradiation $[22,23]$. Although the best results are obtained when PET or CT scan substitutes for surgical staging, the final results of imaging are not yet satisfactory for formulating a proper management plan, especially when it comes to determining the radiation field. Vadeperre et al. demonstrated (analyzing 336 patients with LACC in FIGO stages IB2-IVA) that surgical staging in patients with negative radiologic staging, without any obvious features of metastases in PET or CT imaging, revealed metastases in $8 \%$ of cases. Additionally, surgical staging has a positive effect on overall survival rates in these patients (negative in imaging, but still positive in surgery) [24]. Furthermore, Zand et al. prospectively examined patients with LACC (IB2-IVA) diagnosed through biopsy, and later treated by radiotherapy and concurrent chemotherapy, without any symptoms of lymph node metastases in CT or NMR imaging. All of them underwent PET or CT and laparoscopic para-aortic lymphadenectomy. $22 \%$ of the patients had PA metastases. The radiology imaging correlates to surgical staging. Only one patient had metastases not revealed in PET or CT scan (2\%) [13]. Contrary to this opinion, Yildirim et al. have suggested that PET or CT does enable diagnosing lymph node metastases in the para-aortic area and is an effective imaging technique in LACC. The specificity and sensitivity of PET or CT scans to evaluate the lymph node area for detecting metastatic localization in LACC patients ranges from $50 \%$ to $92 \%$, and from $57 \%$ to $100 \%$, respectively [25]. Lee et al. have suggested that surgical staging of para-aortic nodes are cost-effective methods, while a PET or CT scan does not reveal the presence of lymph node metastases in LACC patients. In $10 \%$ of cases, the para-aortic nodes are involved, even though the PET or CT scan is negative $[22,26]$.

New studies are needed to determine the clinical impact of para-aortic node micrometastases in patients with locally advanced cervical cancer. At the moment, it can be stated that the randomized trial focused to determine whether pre-therapeutic laparoscopic surgical staging followed by tailored chemoradiation impacts survival more than PET or CT radiologic staging alone followed by chemoradiation [27]. Because elderly women with cervical cancer are treated not as radically as younger patients, there is a need to focus the researches on this group. The differences in the therapy depends of the age, starting from 50 years old's (lesser amount of surgery, followed by adjuvant radiation, or brachytherapy) [28]. Older women with post-operative risk factors receiving RT alone experienced similar survival as those undergoing CRT (chemoradiotherapy) [29]. Chopra et al. observed that the patients aged over 70 years had a significantly poor DFS at 5 years; however, did not have any effect on survival [30]. In our study, we have found better survival rates in patients older than 62 years.

\section{Conclusions}

Overall, para-aortic lymphadenectomy seems to have a positive influence on long-term outcomes in LACC patients. In such patients, radical surgery is still a treatment option comparable to radical chemoradiation. We have found that elderly patients may benefit more from the applied therapy.

\section{Disclosure}

Authors report no conflict of interest.

\section{References}

1. Chantalat E, Vidal F, Leguevaque $P$ et al. Cervical cancer with paraaortic involvement: do patients truly benefit from tailored chemoradiation therapy? A retrospective study on 8 French centers. Eur J Obstet Gynecol Reprod Biol 2015; 193: 118-122.

2. Chantalat E, Vidal F, Leguevaque P et al. Para-aortic workup in locally advanced cervical cancer: heterogeneity is still the rule. Results from a retrospective multicenter study. Arch Gynecol Obstet 2016; 293: 1081-1086.

3. Choi HJ, Roh JW, Seo SS et al. Comparison of the accuracy of magnetic resonance imaging and positron emission tomography/computed tomography in the presurgical detection of lymph node metastases in patients with uterine cervical carcinoma: a prospective study. Cancer 2006; 106: 914-922.

4. Margulies AL, Peres A, Barranger E et al. Selection of patients with advanced-stage cervical cancer for para-aortic lymphadenectomy in the era of PET/CT. Anticancer Res 2013; 33: 283-286.

5. Derks M, Biewenga $P$, van der Velden J et al. Results of radical surgery in women with stage IB2/IIA2 cervical cancer. Acta Obstet Gynecol Scand 2016; 95: 166-172.

6. Köhler C, Mustea A, Marnitz S et al. Perioperative morbidity and rate of upstaging after laparoscopic staging for patients with locally advanced cervical cancer: results of a prospective randomized trial. Am J Obstet Gynecol 2015; 213: 503.

7. Zanagnolo V, Minig L, Rollo D et al. Clinical and oncologic outcomes of robotic versus abdominal radical hysterectomy for women with cervical cancer: experience at a Referral Cancer Center. Int J Gynecol Cancer 2016; 26: 568-574.

8. Zhou G, Chen X, Tang F et al. The value of diffusion-weighted imaging in predicting the prognosis of stage IB-IIA cervical squamous cell carcinoma after radical hysterectomy. Int J Gynecol Cancer 2016; 26: 361-366.

9. Macdonald OK, Chen J, Dodson M et al. Prognostic significance of histology and positive lump node involvement following radical hysterectomy in carcinoma of the cervix. Am J Clin Oncol 2009; 32: 411-416.

10. Ouldamer L, Fichet-Djavadian S, Marret $\mathrm{H}$ et al. Upper margin of para-aortic lymphadenectomy in cervical cancer. Acta Obstet Gynecol Scand 2012; 91: 893-900.

11. Ouldamer L, Marret H, Acker O et al. Unusual localizations of sentinel lymph nodes in early stage cervical cancer: a review. Surg Oncol 2012; 21: e153-157.

12. Del Pino M, Fusté P, Pahisa J et al. Laparoscopic lymphadenectomy in advanced cervical cancer: prognostic and therapeutic value. Int J Gynecol Cancer 2013; 23: 1675-1683. 
13. Zand B, Euscher ED, Soliman PT et al. Rate of para-aortic lymph node micrometastasis in patients with locally advanced cervical cancer. Gynecol Oncol 2010; 119: 422-435.

14. Goff BA, Muntz HG, Paley PJ et al. Impact of surgical staging in women with locally advanced cervical cancer. Gynecol Oncol 1999; 74: 436-442.

15. Leblanc E, Katdare N, Narducci F et al. Should systematic infrarenal para-aortic dissection be the rule in the pretherapeutic staging of primary or recurrent locally advanced cervix cancer patients with a negative preoperative para-aortic PET imaging? Int J Gynecol Cancer 2016; 26: 169-175.

16. Kang S, Kim YS, Choi HJ et al. Additional value of combined evaluation of tumor size with lymph node size in the detection of lymph node metastases in early-stage cervical cancer patients. J Comput Assist Tomogr 2013; 37: 572-576.

17. Gil-Moreno A, Magrina JF, Pérez-Benavente A et al. Location of aortic node metastases in locally advanced cervical cancer. Gynecol Oncol 2012; 125: 312-314.

18. Morice P, Castaigne D, Pautier P et al. Interest of pelvic and paraaortic lymphadenectomy in patients with stage IB and II cervical carcinoma. Gynecol Oncol 1999; 73: 106-110.

19. Cosin JA, Fowler JM, Chen MD et al. Pretreatment surgical staging of patients with cervical carcinoma: the case for lymph node debulking. Cancer 1998; 82: 2241-2248.

20. Leblanc E, Narducci F, Frumovitz M et al. Therapeutic value of pretherapeutic extraperitoneal laparoscopic staging of locally advanced cervical carcinoma. Gynecol Oncol 2007; 105: 304-311.

21. Zikan M, Fischerova D, Pinkavova I et al. A prospective study examining the incidence of asymptomatic and symptomatic lymphoceles following lymphadenectomy in patients with gynecological cancer. Gynecol Oncol 2015; 137: 291-298.

22. Lee JY, Kim Y, Lee TJ et al. Cost-effectiveness of para-aortic lymphadenectomy before chemoradiotherapy in locally advanced cervical cancer. J Gynecol Oncol 2015; 26: 171-178.

23. Lee JY, Kwon JS, Cohn DE et al. Treatment strategies for stage IB cervical cancer: A cost-effectiveness analysis from Korean, Canadian and U.S. perspectives. Gynecol Oncol 2016; 140: 83-89.

24. Vandeperre A, van Limbergen E, Leunen K et al. Para-aortic lymph node metastases in locally advanced cervical cancer: Comparison between surgical staging and imaging. Gynecol Oncol 2015; 138: 299-303.

25. Yildirim Y, Sehirali S, Avci ME et al. Integrated PET/CT for the evaluation of para-aortic nodal metastasis in locally advanced cervical cancer patients with negative conventional CT findings. Gynecol Oncol 2008; 108: 154-159.

26. Roh JW, Seo SS, Lee $S$ et al. Role of positron emission tomography in pretreatment lymph node staging of uterine cervical cancer: a prospective surgicopathologic correlation study. Eur J Cancer 2005; 41: 2086-2092.

27. Frumovitz M, Querleu D, Gil-Moreno A et al. Lymphadenectomy in locally advanced cervical cancer study (LiLACS): Phase III clinical trial comparing surgical with radiologic staging in patients with stages IB2-IVA cervical cancer. J Minim Invasive Gynecol 2014; 21: 3-8.

28. Sharma C, Deutsch I, Horowitz DP et al. Patterns of care and treatment outcomes for elderly women with cervical cancer. Cancer 2012; 118: 3618-3626.

29. Cushman TR, Haque W, Menon $\mathrm{H}$ et al. Postoperative chemoradiotherapy versus radiotherapy alone for elderly cervical cancer patients with positive margins, lymph nodes, or parametrial invasion. J Gynecol Oncol 2018; 29 : e97.

30. Chopra S, Gupta M, Mathew A et al. Locally advanced cervical cancer: A study of 5-year outcomes. Indian J Cancer 2018; 55: 45-49. 